International Journal of Pure and Applied Mathematics

Volume 108 No. $4 \quad 2016,865-870$

ISSN: 1311-8080 (printed version); ISSN: 1314-3395 (on-line version)

url: http://www.ijpam.eu

doi: 10.12732/ijpam.v108i4.12

\title{
A NOTE ON WAVELET PACKETS
}

\author{
J. Cheshmavar \\ Department of Mathematics \\ College of Sciences \\ Payame Noor University \\ P.O. Box 19395-4697, Tehran, IRAN
}

\begin{abstract}
The construction of wavelet packets was studied by Chui et. al. in (SIAM J. Math. Anal. 24 (3) 1993, 712-738). In this paper, for any integer dilation factor $p \geq 2$, we construct a orthogonal $p$-wavelet packets based on the Walsh-Fourier transform.
\end{abstract}

AMS Subject Classification: $42 \mathrm{C} 40$

Key Words: $p$-wavelet packets, walsh-Fourier transform, multiresolution analysis

\section{Introduction}

The theory of wavelet packet analysis is an important generalization of wavelet analysis. Shen [7] generalized the notion of univariate orthogonal wavelet packets to the case of multivariate wavelet packets. The orthogonal multi wavelet packets [1]. Farkov [4] constructed orthogonal compactly supported $p$-wavelets on $\mathbb{R}_{+}$. Shah et al. provided a method for constructing wavelet packet on $p$-adic field in [6]. Recently, Chen and Chang [2] investigated the construction of a class of orthogonal vector-valued wavelet packets. Xiao-Feng et al. [5] gave the construction and characterization of vector-valued multivariate wavelet packets associated with dilation matrix by means of time-frequency analysis method, matrix theory and operator theory. In this work, we consider Walsh-Fourier transform and we shall investigate construction of $p$-wavelet packets with and

Received: January 2, 2016

Published: August 29, 2016 (c) 2016 Academic Publications, Ltd.

url: www.acadpubl.eu 
integer dilation factor $p \geq 2$ and construct orthonormal wavelet packet bases of space $L^{2}\left(\mathbb{R}_{+}\right)$based generalized Walsh functions.

\section{Preliminaries and Notations}

Analogue of all the definitions and properties in the following can also be found in $[3,4]$. Denote by $[x]$ the integer part of $x$. For $x$ in $\mathbb{R}_{+}$and any positive integer $j$, we set

$$
x_{j}=\left[p^{j} x\right] \quad(\bmod p), \quad x_{-j}=\left[p^{1-j} x\right] \quad(\bmod p) .
$$

Consider on $\mathbb{R}_{+}$the addition defined as follows: for $x$ and $y$ if $z=x \oplus y$ then

$$
z=\sum_{j<0} \zeta_{j} p^{-j-1}+\sum_{j>0} \zeta_{j} p^{-j}
$$

with

$$
\zeta_{j}=x_{j}+y_{j} \quad(\bmod p) \quad(j \in \mathbb{Z})
$$

where $\zeta_{j} \in\{0,1, \cdots, p-1\}$ and $x_{j}, y_{j}$ are calculated by (2.1). As usual, the equality $z=x \ominus y$ means that $z \oplus y=x$.

For $x \in[0,1)$, let $r_{0}(x)$ be given by

$$
r_{0}(x)= \begin{cases}1, & x \in[0,1 / p) \\ \varepsilon_{p}^{l}, & x \in\left[l p^{-1},(l+1) p^{-1}\right) \quad(l=1, \cdots, p-1)\end{cases}
$$

where $\varepsilon_{p}=\exp (2 \pi i / p)$. The extension of the function $r_{0}$ to $\mathbb{R}_{+}$is denoted by the equality $r_{0}(x+1)=r_{0}(x), x \in \mathbb{R}_{+}$. Then the generalized Walsh functions $\left\{w_{m}(x): m \in \mathbb{Z}_{+}\right\}$are defined by

$$
w_{0}(x) \equiv 1, \quad w_{m}(x)=\prod_{j=0}^{k}\left(r_{0}\left(p^{j} x\right)\right)^{\mu_{\mathrm{j}}}
$$

where

$$
m=\sum_{j=0}^{k} \mu_{j} p^{j}, \quad \mu_{j} \in\{0,1, \ldots, p-1\}, \quad \mu_{k} \neq 0 .
$$

For $x, w \in \mathbb{R}_{+}$, we set 


$$
\chi(x, w)=\exp \left(\frac{2 \pi i}{p} \sum_{j=1}^{\infty}\left(x_{j} w_{-j}+x_{-j} w_{j}\right)\right)
$$

where $x_{j}, w_{j}$ are given by (2.1). The Walsh-Fourier transform of a function $f \in L^{1}\left(\mathbb{R}_{+}\right)$is defined by

$$
\hat{f}(\xi)=\int_{\mathbb{R}_{+}} f(x) \overline{\chi(x, \xi)} d x,
$$

where $\chi(x, \xi)$ is given by $(2.2)$. The properties of the Walsh-Fourier transform are similar to the Fourier transform. Particular, if $f \in L^{2}\left(\mathbb{R}_{+}\right)$, then $\hat{f} \in$ $L^{2}\left(\mathbb{R}_{+}\right)$and

$$
\|\hat{f}\|_{L^{2}\left(\mathbb{R}_{+}\right)}=\|f\|_{L^{2}\left(\mathbb{R}_{+}\right)} .
$$

Also, the inverse formula is the form

$$
f(x)=\int_{\mathbb{R}_{+}} \hat{f}(x) \overline{\chi(x, \xi)} d \xi
$$

We recall that the definition of multiresolution $p$-analysis in $L^{2}\left(\mathbb{R}_{+}\right)$.

Definition 2.1. A sequence $\left\{V_{j}: j \in \mathbb{Z}\right\}$ of closed subspaces of $L^{2}\left(\mathbb{R}_{+}\right)$ is called a vector-valued multiresolution $p$-analysis of $L^{2}\left(\mathbb{R}_{+}\right)$, if the following conditions are satisfied:

(i) $V_{j} \subset V_{j+1}, \forall j \in \mathbb{Z}$;

(ii) $\bigcap_{j \in \mathbb{Z}} V_{j}=\{0\}$, and $\bigcup_{j \in \mathbb{Z}} V_{j}$ is dense in $L^{2}\left(\mathbb{R}_{+}\right)$;

(iii) $f(\cdot) \in V_{j}$ if and only if $f(p \cdot) \in V_{j+1}, \forall j \in \mathbb{Z}$;

(iv) There exists a function $\varphi \in V_{0}$, called the scaling function, such that $\left\{\varphi_{k}(x):=\varphi(\cdot \ominus k): k \in \mathbb{Z}_{+}\right\}$constitute a orthogonal basis for $V_{0}$.

Since $\varphi \in V_{-1} \subset V_{0}$, there exist a sequence $\left\{P_{k}: k \in \mathbb{Z}_{+}\right\}$with $\sum k \in \mathbb{Z}_{+}\left|P_{k}\right|^{2}$ $<\infty$ such that

$$
p^{-1} \varphi\left(x p^{-1}\right)=\sum_{k \in \mathbb{Z}_{+}} P_{k} \varphi(x \ominus k) .
$$




\section{Orthogonal $p$-Wavelet Packets}

Let $W_{0}$ be the orthogonal complement of $V_{0}$ to $V_{1}$, i.e., $V_{1}=V_{0} \oplus W_{0}$. If there exists $\left\{\psi_{1}, \cdots \psi_{p-1}\right\}$ such that $\left\{\psi_{l}(\cdot \ominus k): 1 \leq l \leq p, k \in \mathbb{Z}_{+}\right\}$forms an orthonormal basis for $W_{0}$. We call $\left\{\psi_{1}, \cdots \psi_{p-1}\right\}$ an orthogonal p-wavelet associated with $\varphi(x)$.

From $p^{-1} \psi_{l}(\cdot / p) \in W_{-1} \subset V_{0}$, there exists a sequence $\left\{Q_{k}: k \in \mathbb{Z}_{+}\right\}$with $\sum_{k \in \mathbb{Z}_{+}}\left|Q_{k}\right|^{2}<\infty$ such that

$$
p^{-1} \psi_{l}\left(x p^{-1}\right)=\sum_{k \in \mathbb{Z}_{+}} Q_{k}^{l} \psi(x \ominus k)
$$

set

$$
P(\xi)=\sum_{k \in \mathbb{Z}_{+}} P_{k} \overline{\chi(k, \xi)} \quad \text { and } \quad Q_{l}(\xi)=\sum_{k \in \mathbb{Z}_{+}} Q_{k} \overline{\chi(k, \xi)}
$$

By taking Walsh-Fourier transform, we get

$$
\hat{\varphi}(\xi p)=P(\xi) \hat{\varphi}(\xi) \quad \text { and } \quad \hat{\psi}_{l}(\xi p)=Q_{l}(\xi) \hat{\varphi}(\xi)
$$

$P(\xi)$ and $Q(\xi)$ are refinement mask and wavelet mask, respectively.

Lemma 3.1. for $\varphi \in L^{2}\left(\mathbb{R}_{+}\right),\left\{\varphi(\cdot \ominus k): k \in \mathbb{Z}_{+}\right\}$are orthonormal in $L^{2}\left(\mathbb{R}_{+}\right)$if and only if

$$
\sum_{l \in \mathbb{Z}_{+}}|\hat{\varphi}(\xi+l)|^{2}=1 \text { a.e } \xi \in \mathbb{R}_{+}
$$

Proof. We have

$$
\begin{aligned}
\int_{\mathbb{R}_{+}} \varphi(x) \overline{\varphi(x \ominus k)} d x & =\int_{\mathbb{R}_{+}}|\hat{\varphi}(\xi)|^{2} \overline{\chi(k, \xi)} d \xi \\
& =\sum_{l=0}^{\infty} \int_{l}^{l+1}|\hat{\varphi}(\xi)|^{2} \overline{\chi(k, \xi)} d \xi \\
& =\int_{0}^{1}\left(\sum_{l=0}^{\infty}|\hat{\varphi}(\xi+l)|^{2}\right) \overline{\chi(k, \xi)} d \xi
\end{aligned}
$$

Therefor, $\left\{\varphi(\cdot \ominus k): k \in \mathbb{Z}_{+}\right\}$are orthonormal if and only if $\sum_{l \in \mathbb{Z}_{+}}|\hat{\varphi}(\xi+l)|^{2}=$ 1 a.e $\xi \in \mathbb{R}_{+}$ 
Now let $P_{k}^{i}$ be a high-pass filter sequence associated to $\psi_{i}(x), i=1, \cdots p-1$. Denote $U_{0}(x)=\varphi(x)$, and $U_{i}(x)=\psi_{i}(x), i=1, \cdots p-1$. Then the two scaling equation can be written as

$$
U_{0}(x)=\sum_{k \in \mathbb{Z}_{+}} P_{k}^{0} U_{0}(p x \ominus k) \text { and } U_{i}(x)=\sum_{k \in \mathbb{Z}_{+}} P_{k}^{i} U_{0}(p x \ominus k) i=1, \cdots p-1
$$

orthogonal p-wavelet packets with respect to $\varphi(x)$ are defined by

$$
U_{p l+i}(x)=\sum_{k \in \mathbb{Z}_{+}} P_{k}^{i} U_{l}(p x \ominus k) .
$$

where $i=0, \cdots p-1, l=0,1, \cdots$. Note that (3.4) defines $U_{n}$ for all $n \geq 0$. Taking Walsh-Fourier transform in both side of (3.4), we get

$$
\left(U_{p l+i}\right)^{\wedge}(\xi)=Q_{i}\left(p^{-1} \xi\right) \hat{\omega}_{l}\left(p^{-1} \xi\right), \quad i=0, \cdots p-1 .
$$

The functions will be called the basic p-wavelet packets associated with multiresolution p-analysis. For $n=0,1, \cdots$, in order to describe the Fourier transform of $U_{p l+i}$, we need write $n$ in the form of $n=\sum_{j=0}^{k} \epsilon_{j} p^{j}, \epsilon_{j} \in$ $\{0,1, \cdots p-1\}, \epsilon_{k} \neq 0, k \in \mathbb{Z}_{+}$.

Theorem 3.2. Let $\left\{U_{n}: n \geq 0\right\}$ be the basic p-wavelet packets constructed above and the unique expansion of $n$ be as the above. Then

$$
\hat{U}_{n}(\xi)=\prod_{j=0}^{k} Q_{\epsilon_{\mathrm{j}}}\left(p^{-j} \xi\right) \hat{\varphi}\left(p^{-k} \xi\right)
$$

Proof. For arbitrary non-negative integer $n$, we show it by induction. If the length of $n$ is equal 1, clearly (3.6) hold. Assume that it true for all $n$ of length $k$. Then

$$
\begin{aligned}
\hat{U}_{\epsilon+p n} & =Q_{\epsilon}\left(p^{-1 \xi}\right) \hat{U}_{n}\left(p^{-1 \xi}\right) \\
& =\prod_{j=0}^{k+1} Q_{\epsilon_{\mathrm{j}}}\left(p^{-j} \xi\right) \hat{\varphi}\left(p^{-(k+1)} \xi\right)
\end{aligned}
$$

\section{References}

[1] B. Behera, Multiwavelet packets and frame packets of $L^{2}\left(\mathbb{R}^{d}\right)$, Proc. Indian Acad. Sci. Math. Sci., 111 (2001), 439-463. 
[2] Q. Chen, Z. Chang, A study on compactly supported orhogonal vector-valued wavelets and wavelet packets, Chaos Solitons Fractals, 31 (2007), 1024-1034.

[3] Yu.A. Farkov, Orthogonal $p$-wavelets on $\mathbb{R}_{+}$, Proc. Int. Conf. Wavelets and Splines, St. Petersburg Univ. Press (2005), 4-26.

[4] Yu.A. Farkov, Orthogonal wavelets on direct products of cyclic groups, Math. Notes, 82, No. 6 (2007), 843-859.

[5] W.X. Feng, H. Gao, J. Feng, The characterization of vector-valued multivariate wavelet packets associated with a dilation matrix, Chaos Solitons Fractals, 42 (2009), 1959-1966.

[6] F.A. Shah, Construction of wavelet packets on p-adic fild, Int. J. Wavelets Multi. Inf. Proc., 7, No. 5 (2009), 553-565.

[7] Z. Shen, Non-tensor product wavelet packets in $L^{2}\left(\mathbb{R}^{s}\right)$, SIAM J. Math. Anal., 26, No. 4 (1995), 1061-1074. 